\title{
A NOTE ON FINITE ABELIAN GROUPS ${ }^{1}$
}

\section{J. PAIGE}

1. Introduction. R. H. Bruck ${ }^{2}$ has pointed out that every finite group of odd order is isotopic to an idempotent quasigroup. It can be shown that a necessary and sufficient condition that a group $G$ be isotopic to an idempotent quasigroup is that there exist one-to-one mappings $\theta$ and $\eta$ of $G$ upon $G$ satisfying the relationship $\eta(x)=x \cdot \theta(x)$, for all $x$ of $G$. The same condition is sufficient to prove the existence of a loop $M$ whose automorphism group contains $G$ as a subgroup. We shall not attempt to show either of these applications; but, since there may be others, the present paper is concerned with the existence of suitable $\theta$ and $\eta$ for any finite abelian group $G$. For this we have a complete answer. Our methods are constructive, but (unfortunately from the standpoint of generalization) they make considerable use of the commutative law.

2. Notation. We shall consider a finite abelian group $G$ of order $n=n(G)$.

The product of the $n$ distinct elements of $G$ will be designated by $p=p(G)$.

Let $x \rightarrow \theta(x)$ be any one-to-one mapping (not necessarily an automorphism) of $G$ upon $G$. Consider the derived mapping $x \rightarrow \eta(x)$ $=x \theta(x)$. The order of $\eta$, denoted by $O(\eta)$, is the number of distinct elements $\eta(x)$, for $x$ in $G$.

It is our purpose to prove the following theorem:

THEOREM 1. There exists a $\theta$ for which $O(\eta)=n(G)$ unless $G$ possesses exactly one element of order 2 . In the latter case there exists $a \theta$ for which $O(\eta)=n(G)-1$.

\section{Evaluation of $p$.}

Lemma 1. $p(G)=1$ unless $G$ possesses exactly one element of order 2 . In the latter case, $p(G)$ is the unique element of order 2.

Proof. The set $H$ consisting of the identity and all elements of $G$ of order 2 is a uniquely defined subgroup of $G$. If $a \in G$ is of order

Presented to the Society, November 30,1946; received by the editors October 17, 1946, and, in revised form, December 5, 1946.

${ }^{1}$ The author wishes to thank the referee for his suggestions which added considerably to the clarity of the paper.

2 R. H. Bruck, Some results in the theory of quasigroups, Trans. Amer. Math. Soc. vol. 55 (1944) pp. 19-52, especially pp. $35,36$. 
greater than 2, $a \neq a^{-1}$; thus both $a$ and $a^{-1}$ appear in $p(G)$ and hence $p(G)=p(H)$.

If $H$ has order $1, p(H)=1$. If $H$ has order 2 , elements $1, g$, then $p(H)=1 \cdot g=g$, and $p(H)$ is the unique element of $H$ (and hence of $G$ ) of order 2 .

Now suppose $H$ has order greater than 2; so that $H$ has order $2^{k}$, $k>1$. Then $H$ has $k$ generators $g_{1}, \cdots, g_{k}$ and every element of $H$ has a unique representation in the form $g_{1}^{n_{1}} g_{2}^{n_{2}} \cdots g_{k}^{n_{k}}$ where $n_{i}$ is 0 or 1 . Hence $p(H)=\prod\left(g_{1}^{n_{1}} g_{2}^{n_{2}} \cdots g_{k}^{n_{k}}\right)$, where the product is over the distinct ordered sets $\left(n_{1}, \cdots, n_{k}\right)$ with $n_{i}$ taking the values 0 or 1 . By symmetry $p(H)=\left(g_{1} g_{2} \cdots g_{k}\right)^{m}$ where $m=2^{k-1}$ and since $k>1$ we have $p(H)=1$.

4. A necessary condition. It is easily shown that there are abelian groups for which a suitable $\theta$ does not exist.

Lemma 2. A necessary condition that $O(\eta)=n(G)$ is that $p(G)=1$.

Corollary. If $p(G) \neq 1, O(\eta)<n(G)$ for all $\theta$.

Proof. Suppose there exists a $\theta$ for which $O(\eta)=n(G)$. Then if we denote the elements of $G$ by $x_{i}(i=1,2, \cdots, n)$,

$$
\prod_{i=1}^{n}\left[x_{i} \theta\left(x_{i}\right)\right]=\prod_{i=1}^{n} \eta\left(x_{i}\right),
$$

and since $G$ is abelian, $\theta$ and $\eta$ one-to-one mappings of $G$ upon $G$, we have $p^{2}=p$ or $p=1$. The corollary should be obvious.

5. The main theorem. In order to avoid complexity, we prove the following lemma before proceeding with the proof of Theorem 1.

LEMMA 3. If for $\theta, O(\eta) \leqq n-2$, where $n=n(G)$, there exists a $\theta^{\prime}$ such that $O\left(\eta^{\prime}\right)>O(\eta)$.

CoRollaRy. There exists a $\theta$ for which $O(\eta)=n(G)-1$.

Proof. Let $\theta$ be a mapping for which $O(\eta)=r \leqq n-2$. Denoting the elements of $G$ by $x_{i}(i=1, \cdots, n)$, let $\eta\left(x_{i}\right)(i=1, \cdots, r)$ be the $r$ distinct elements of $\eta(x)$, for $x$ in $G$. If there exist integers $h, k>r$ such that $x_{h} \theta\left(x_{k}\right) \neq \eta\left(x_{i}\right)(i \leqq r)$, the problem is solved by setting $\theta^{\prime}\left(x_{h}\right)=\theta\left(x_{k}\right), \theta^{\prime}\left(x_{k}\right)=\theta\left(x_{h}\right)$ and $\theta^{\prime}(x)=\theta(x)$ for the remaining elements of $G$. Hence, assume that this is not the case. Since $\eta\left(x_{r+1}\right)$ $=\eta\left(x_{i}\right)$ for some $i \leqq r$, there is no loss in generality in assuming that

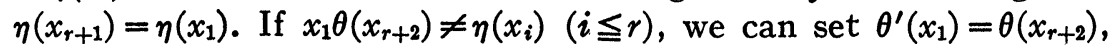
$\theta^{\prime}\left(x_{r+2}\right)=\theta\left(x_{1}\right)$ leaving $\theta^{\prime}(x)=\theta(x)$ for the remaining elements of $G$ 
and thus construct a $\theta^{\prime}$ with $O\left(\eta^{\prime}\right)>r$. But if $x_{1} \theta\left(x_{r+2}\right)=\eta\left(x_{i}\right)$ for some $i \leqq r$, we note that $x_{1} \theta\left(x_{r+2}\right) \neq \eta\left(x_{1}\right)$. Hence we may assume without loss of generality that $x_{1} \theta\left(x_{r+2}\right)=\eta\left(x_{2}\right)$.

Now $x_{2} \theta\left(x_{1}\right) \neq \eta\left(x_{1}\right), \eta\left(x_{2}\right)$. If $x_{2} \theta\left(x_{1}\right) \neq \eta\left(x_{i}\right)(i \leqq r)$, we could change $\theta$ by setting $\theta^{\prime}\left(x_{1}\right)=\theta\left(x_{r+2}\right), \theta^{\prime}\left(x_{2}\right)=\theta\left(x_{1}\right), \theta^{\prime}\left(x_{r+2}\right)=\theta\left(x_{2}\right)$ and thus construct $\theta^{\prime}$ with $O\left(\eta^{\prime}\right)>r$. Otherwise we may assume without loss of generality that $x_{2} \theta\left(x_{1}\right)=\eta\left(x_{3}\right)$.

Continue in this manner and suppose we have reached the point where

$$
x_{1} \theta\left(x_{r+2}\right)=\eta\left(x_{2}\right), \quad x_{i+1} \theta\left(x_{i}\right)=\eta\left(x_{i+2}\right) \quad(i=1,2, \cdots, k) .
$$

From (1) we derive the equations

$$
\eta\left(x_{1}\right) \theta\left(x_{r+2}\right)=\eta\left(x_{i+1}\right) \theta\left(x_{i}\right) \quad(i=1,2, \cdots, k+1) .
$$

In fact $\eta\left(x_{1}\right) \theta\left(x_{r+2}\right)=x_{1} \theta\left(x_{1}\right) \theta\left(x_{r+2}\right)=x_{1} \theta\left(x_{r+2}\right) \theta\left(x_{1}\right)=\eta\left(x_{2}\right) \theta\left(x_{1}\right)$; so assume $\eta\left(x_{1}\right) \theta\left(x_{r+2}\right)=\eta\left(x_{j+1}\right) \theta\left(x_{j}\right)$ for some $j$, with $1 \leqq j \leqq k$. Then $\eta\left(x_{j+1}\right) \theta\left(x_{j}\right)=x_{j+1} \theta\left(x_{j}\right) \theta\left(x_{j+1}\right)=\eta\left(x_{j+2}\right) \theta\left(x_{j+1}\right)$; and the result follows by induction.

Now $x_{k+2} \theta\left(x_{k+1}\right) \neq \eta\left(x_{i}\right)(i \leqq k+2)$, for using (2) this would imply $\eta\left(x_{i}\right) \theta\left(x_{k+2}\right)=x_{k+2} \theta\left(x_{k+1}\right) \theta\left(x_{k+2}\right)=\eta\left(x_{k+2}\right) \theta\left(x_{k+1}\right)=\eta\left(x_{i}\right) \theta\left(x_{i-1}\right)$, or $\theta\left(x_{k+2}\right)=\theta\left(x_{i-1}\right)$, which is impossible since $i \leqq k+2$. If $x_{k+2} \theta\left(x_{k+1}\right)$ $\neq \eta\left(x_{i}\right)$ (i@r), we could change $\theta$ by setting $\theta^{\prime}\left(x_{1}\right)=\theta\left(x_{r+2}\right), \theta^{\prime}\left(x_{i+1}\right)$ $=\theta\left(x_{i}\right)(i=1,2, \cdots, k+1), \theta^{\prime}\left(x_{r+2}\right)=\theta\left(x_{k+2}\right)$ and thus construct a $\theta^{\prime}$ with $O\left(\eta^{\prime}\right)>r$. If $x_{k+2} \theta\left(x_{k+1}\right)=\eta\left(x_{i}\right)$ for some $i \leqq r$ we may assume without loss of generality that $i=k+3$ and add to (1) the equation $x_{k+2} \theta\left(x_{k+1}\right)=\eta\left(x_{k+3}\right)$. However, since $O(\eta)$ is finite, we must reach a product $x_{j} \theta\left(x_{j-1}\right) \neq \eta\left(x_{i}\right)(i \leqq r)$. This completes the proof of Lemma 3. The corollary is obvious.

In order to prove Theorem 1 we may assume, by the corollary of Lemma 3, a $\theta$ for which $O(\eta)=n(G)-1$. Hence, let $\eta\left(x_{i}\right)(i=1, \cdots$, $n-1)$ be the $n-1$ distinct elements of $\eta(x)$, for $x$ in $G ; z$ the unique element of $G$ not equal to some $\eta\left(x_{i}\right)$. Then since

$$
\prod_{i=1}^{n-1}\left[x_{i} \theta\left(x_{i}\right)\right]=\prod_{i=1}^{n-1} \eta\left(x_{i}\right)
$$

we have $p x_{n}^{-1} p \theta\left(x_{n}\right)^{-1}=p z^{-1}$, where $p=p(G)$ as defined in $\S 2$. Thus $p^{-1} x_{n} \theta\left(x_{n}\right)=z$ or $p^{-1} \eta\left(x_{n}\right)=z$. Hence if $p(G)=1$, we see that $O(\eta)$ $=n(G)$. But if $p(G) \neq 1$ we know by Lemma 2 that $O(\eta)<n(G)$ for all $\theta$. This completes the proof.

Although there exist groups $G$ for which a $\theta$, such that $O(\eta)=n(G)$, is easily represented explicitly (for example, if $G$ is of odd order let $\theta(x)=x)$, the author found it necessary to use repeated applications 
of Lemma 3 to obtain suitable $\theta$ 's for groups of the form $Z_{1} \times Z_{2} \times Z_{3}$ where $Z_{i}$ are cyclic of order $2^{n} i$. However, it should be noted that if $G \cong G_{1} \times G_{2}$, a one-to-one mapping $\theta$ of $G$ upon $G$ may be defined by

$$
\theta[(x, y)]=\left[\theta_{1}(x), \theta_{2}(y)\right]
$$

where $\theta_{1}$ and $\theta_{2}$ are one-to-one mappings of $G_{1}$ upon $G_{1}$ and $G_{2}$ upon $G_{2}$ respectively. Moreover $\theta$ satisfies the relationship $O(\eta) \geqq O\left(\eta_{1}\right) \cdot O\left(\eta_{2}\right)$. Thus if $O\left(\eta_{1}\right)=n\left(G_{1}\right), O\left(\eta_{2}\right)=n\left(G_{2}\right)$ we would have $O(\eta)=n\left(G_{1} \times G_{2}\right)$ and $\theta$ is represented explicitly in terms of $\theta_{1}$ and $\theta_{2}$.

UNIVERSITY OF WISCONSIN

\section{ON RINGS WHOSE ASSOCIATED LIE RINGS ARE NILPOTENT}

\section{S. A. JENNINGS}

1. Introduction. With any ring $R$ we may associate a Lie ring $(R)_{l}$ by combining the elements of $R$ under addition and commutation, where the commutator $x \circ y$ of two elements $x, y \in R$ is defined by

$$
x \circ y=x y-y x \text {. }
$$

We call $(R)_{l}$ the Lie ring associated with $R$, and denote it by $\Re$. The question of how far the properties of $\Re$ determine those of $R$ is of considerable interest, and has been studied extensively for the case when $R$ is an algebra, but little is known of the situation in general. In an earlier paper the author investigated the effect of the nilpotency of $\Re$ upon the structure of $R$ if $R$ contains a nilpotent ideal $N$ such that $R / N$ is commutative. ${ }^{1}$ In the present note we prove that, for an arbitrary ring $R$, the nilpotency of $\Re$ implies that the commutators of $R$ of the form $x \circ y$ generate a nil-ideal, while the commutators of $R$ of the form ( $x \circ y) \circ z$ generate a nilpotent ideal (cf. $\S 3$ ). If $R$ is finitely generated, and $\Re$ is nilpotent then the ideal generated by the commutators $x \circ y$ is also nilpotent (cf. $\$ 4$ ).

2. A lemma on $L$-nilpotent rings. We recall that the Lie ring $\Re$ is said to be nilpotent of class $\gamma$ if we have

Received by the editors December 23, 1946.

1 Central chains of ideals in an associative ring, Duke Math. J. vol. 9 (1942) pp. 341-355, Theorem 6.5. 